Bull. Korean Math. Soc. 46 (2009), No. 6, pp. 1201-1211

DOI 10.4134/BKMS.2009.46.6.1201

\title{
APPLICATIONS OF GENERALIZED KUMMER'S SUMMATION THEOREM FOR THE SERIES ${ }_{2} F_{1}$
}

\author{
Yong Sup Kim and ArJun K. Rathie
}

\begin{abstract}
The aim of this research paper is to establish generalizations of classical Dixon's theorem for the series ${ }_{3} F_{2}$, a result due to Bailey involving product of generalized hypergeometric series and certain very interesting summations due to Ramanujan. The results are derived with the help of generalized Kummer's summation theorem for the series ${ }_{2} F_{1}$ obtained earlier by Lavoie, Grondin, and Rathie.
\end{abstract}

\section{Introduction}

The generalized hypergeometric functions with $p$ numerator and $q$ denominator parameters is defined by [7]

$$
\begin{aligned}
{ }_{p} F_{q}\left[\begin{array}{c}
\alpha_{1}, \ldots, \alpha_{p} \\
\beta_{1}, \ldots, \beta_{q}
\end{array} ; z\right] & ={ }_{p} F_{q}\left[\alpha_{1}, \ldots, \alpha_{p} ; \beta_{1}, \ldots, \beta_{q} ; z\right] \\
& :=\sum_{n=0}^{\infty} \frac{\left(\alpha_{1}\right)_{n} \cdots\left(\alpha_{p}\right)_{n}}{\left(\beta_{1}\right)_{n} \cdots\left(\beta_{q}\right)_{n}} \frac{z^{n}}{n !},
\end{aligned}
$$

where $(\alpha)_{n}$ denotes the Pochhammer symbol (or the shifted factorial, since $(1)_{n}=n$ !) defined for any complex number $\alpha$ by

$$
(\alpha)_{n}= \begin{cases}\alpha(\alpha+1) \cdots(\alpha+n-1), & \text { if } n \in \mathbb{N}=\{1,2, \ldots\} \\ 1, & \text { if } n=0\end{cases}
$$

using the fundamental relation $\Gamma(\alpha+1)=\alpha \Gamma(\alpha),(\alpha)_{n}$ can be written in the form

$$
(\alpha)_{n}=\frac{\Gamma(\alpha+n)}{\Gamma(\alpha)} \quad(n \in \mathbb{N} \cup\{0\}),
$$

where $\Gamma$ is the well-known Gamma function.

Received October 2, 2008.

2000 Mathematics Subject Classification. 33C05, 33C20.

Key words and phrases. generalized Kummer's theorem, generalized Dixon's theorem, generalized Whipple's theorem.

This paper was supported by Wonkwang University in 2009.

(C)2009 The Korean Mathematical Society 
It is well-known that whenever a generalized hypergeometric function reduces to the Gamma function, the results are very important from the application point of view. We mention here some of special cases of (1.1).

Gauss's summation theorem [3]:

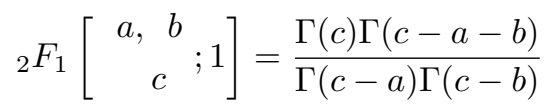

provided $\Re(c-a-b)>0$.

Kummer's summation theorem [3]:

$$
{ }_{2} F_{1}\left[\begin{array}{l}
a, b \\
1+a-b
\end{array} ;-1\right]=\frac{\Gamma\left(\frac{1}{2}\right) \Gamma(1+a-b)}{2^{a} \Gamma\left(\frac{1}{2} a+\frac{1}{2}\right) \Gamma\left(1+\frac{1}{2} a-b\right)} .
$$

Dixon's summation theorem [3]:

$$
\begin{aligned}
& { }_{3} F_{2}\left[\begin{array}{l}
a, b, c \\
1+a-b, 1+a-c
\end{array} ; 1\right] \\
& =\frac{\Gamma\left(1+\frac{1}{2} a\right) \Gamma(1+a-b) \Gamma(1+a-c) \Gamma\left(1+\frac{1}{2} a-b-c\right)}{\Gamma(1+a) \Gamma\left(1+\frac{1}{2} a-b\right) \Gamma\left(1+\frac{1}{2} a-c\right) \Gamma(1+a-b-c)}
\end{aligned}
$$

provided $\Re(a-2 b-2 c)>-2$.

On the other hand, an interesting result due to Bailey [2] involving product of generalized hypergeometric series is

$$
{ }_{0} F_{1}\left[\begin{array}{c}
- \\
\rho
\end{array} x\right] \times{ }_{0} F_{1}\left[\begin{array}{c}
- \\
\rho
\end{array}-x\right]={ }_{0} F_{3}\left[\begin{array}{c}
- \\
\rho, \frac{1}{2} \rho, \frac{1}{2} \rho+\frac{1}{2} ;-\frac{x^{2}}{4}
\end{array}\right] .
$$

The followings are the summations due to Ramanujan [4]:

$$
1-\left(\frac{1}{2}\right)^{2}+\left(\frac{1 \cdot 3}{2 \cdot 4}\right)^{2}-\cdots+(-1)^{n}\left(\frac{\left(\frac{1}{2}\right)_{n}}{n !}\right)^{2}+\cdots=\frac{\sqrt{\pi}}{\sqrt{2} \Gamma^{2}(3 / 4)}
$$

and for $\Re(x)>0$

$$
1+\frac{(x-1)}{(x+1)}+\frac{(x-1)(x-2)}{(x+1)(x+2)}+\cdots+(-1)^{n} \frac{(1-x)_{n}}{(x+1)_{n}}+\cdots=\frac{2^{2 x-1} \Gamma^{2}(x+1)}{\Gamma(2 x+1)} .
$$

In the theory of generalized hypergeometric series, classical summation theorems for the series ${ }_{2} F_{1},{ }_{3} F_{2},{ }_{4} F_{3}$ and ${ }_{5} F_{4}$ play an important role. The Dixon's theorem (1.6) can be obtained with the help of Gauss's summation theorem (1.4) and Kummer's summation theorem (1.5). Bailey [2] derived the result (1.7) by utilizing Kummer's summation theorem (1.7). Berndt [4] pointed out that the summation (1.8) and (1.9) due to Ramanujan can be obtained quite simply from Kummer's theorem (1.5) by taking $a=b=\frac{1}{2}$ and $a=1, b=1-x$, respectively. 
In 1996, Grodin and Rathie [6] generalized the Kummer's theorem (1.5) in the form

(1.10)

$$
\begin{aligned}
& { }_{2} F_{1}\left[\begin{array}{c}
a, b \\
1+a-b+i
\end{array} ;-1\right] \\
= & \frac{\Gamma\left(\frac{1}{2}\right) \Gamma(1+a-b+i) \Gamma(1-b)}{2^{a} \Gamma\left(1-b+\frac{1}{2}(i+|i|)\right)} \\
& \times\left\{\frac{A_{i}}{\Gamma\left(\frac{1}{2} a+\frac{1}{2} i+\frac{1}{2}-\left[\frac{1+i}{2}\right]\right) \Gamma\left(1+\frac{1}{2} a-b+\frac{1}{2} i\right)}+\frac{B_{i}}{\Gamma\left(\frac{1}{2} a+\frac{1}{2} i-\left[\frac{i}{2}\right]\right) \Gamma\left(\frac{1}{2}+\frac{1}{2} a-b+\frac{i}{2}\right)}\right\}
\end{aligned}
$$

for $i=0, \pm 1, \pm 2, \pm 3, \pm 4, \pm 5$.

As usual, $[x]$ denotes the greatest integer less than or equal to $x$ and its modulus is denoted by $|x|$. The coefficients $A_{i}$ and $B_{i}$ are given respectively in $[6$, p. 298]. The paper is organized as follows. In Section 2, explicit expression for the series

$$
{ }_{3} F_{2}\left[\begin{array}{c}
a, b, \quad c \\
1+a-b+i, 1+a-c
\end{array} ; 1\right]
$$

for $i=0, \pm 1, \pm 2, \pm 3, \pm 4, \pm 5$ are given. The results will be derived with the help of generalized Kummer's theorem (1.10) and Gauss's theorem (1.4). As an applications generalizations of certain summations due to Ramanujan are also given. In Section 3, single series expressions of

$$
{ }_{0} F_{1}\left[\begin{array}{c}
- \\
\rho
\end{array} ; x\right] \times{ }_{0} F_{1}\left[\begin{array}{c}
- \\
\rho+i
\end{array} ;-x\right]
$$

for $i=0, \pm 1, \pm 2, \pm 3, \pm 4, \pm 5$ are given. As special cases, some interesting results are also mentioned. In Section 4, summations for the series

$$
1-\left(\frac{1}{2}\right)^{2} \frac{1}{(1+i)} \frac{1}{1 !}+\left(\frac{1 \cdot 3}{2 \cdot 4}\right)^{2} \frac{1}{(1+i)(2+i)} \frac{1}{2 !}-\cdots+(-1)^{n} \frac{\left(\frac{1}{2}\right)_{n}\left(\frac{1}{2}\right)_{n}}{n !(1+i)_{n}}+\cdots
$$

and for $\Re(x)>0$

$$
1+\frac{(x-1)}{(x+1+i)}+\frac{(x-1)(x-2)}{(x+1+i)(x+2+i)}+\cdots+(-1)^{n} \frac{(1-x)_{n}}{(x+1+i)_{n}}+\cdots
$$

each for $i=0,1,2,3,4,5$ are given. The results given in Sections 3 and 4 are derived with the help of generalized Kummer's theorem (1.10).

\section{Generalization of Dixon's theorem}

In this section, the result to be proved is given in the following theorem. 
Theorem 1. For $\Re(a-2 b-2 c+i)>-2$, we have

$$
\begin{aligned}
& { }_{3} F_{2}\left[\begin{array}{c}
a, \quad b, \quad c \\
1+a-b+i, 1+a-c
\end{array} ; 1\right] \\
= & \frac{\Gamma\left(\frac{1}{2}\right) \Gamma(1-b) \Gamma(1+a-b+i)}{\Gamma\left(1-b+\frac{1}{2}(i+|i|)\right)} \sum_{m=0}^{\infty} \frac{\left(\frac{1}{2} a\right)_{m}\left(\frac{1}{2} a+\frac{1}{2}\right)_{m}\left(b-\frac{1}{2}(i+|i|)\right)_{m}}{(1+a-c)_{m}} \\
& \times\left\{\frac{A_{i}{ }^{\prime}}{\Gamma\left(\frac{1}{2} a-b+\frac{1}{2} i+1\right) \Gamma\left(\frac{1}{2} a+\frac{1}{2}+\frac{1}{2} i-\left[\frac{1+i}{2}\right]\right)\left(\frac{1}{2} a+\frac{1}{2}+\frac{1}{2} i-\left[\frac{1+i}{2}\right]\right)_{m}}\right. \\
& \left.+\frac{B_{i}{ }^{\prime}}{\Gamma\left(\frac{1}{2} a-b+\frac{1}{2} i+\frac{1}{2}\right) \Gamma\left(\frac{1}{2} a+\frac{1}{2} i-\left[\frac{i}{2}\right]\right)\left(\frac{1}{2} a+\frac{1}{2}-\left[\frac{i}{2}\right]\right)_{m}}\right\}
\end{aligned}
$$

for $i=0, \pm 1, \pm 2, \pm 3, \pm 4, \pm 5$. The coefficients $A_{i}{ }^{\prime}$ and $B_{i}{ }^{\prime}$ can be obtained from the tables of $A_{i}$ and $B_{i}$ by changing $a$ by $a+2 m$ and $b$ by $b+m$ respectively in [6, p. 298].

Proof. Denoting the left-hand side by $\mathcal{L}$, we have

$$
\mathcal{L}={ }_{3} F_{2}\left[\begin{array}{c}
a, b, c \\
1+a-b+i, 1+a-c
\end{array} ; 1\right] .
$$

Expressing as a series,

$$
\mathcal{L}=\sum_{k=0}^{\infty} \frac{(a)_{k}(b)_{k}(c)_{k}}{(1+a-b+i)_{k}(1+a-c)_{k} k !} .
$$

We can write this in the form

$$
=\sum_{k=0}^{\infty} \frac{(a)_{k}(b)_{k}}{(1+a-b+i)_{k}(-1)_{k} k !}\left\{\frac{(-1)^{k}(c)_{k}}{(1+a-c)_{k}}\right\} .
$$

Using a result $[7$, p. 69, Ex. 5]

$$
\begin{gathered}
{ }_{2} F_{1}\left[\begin{array}{cc}
-n, & a+n \\
b & ; 1
\end{array}\right]=\frac{(-1)^{n}(1+a-b)_{n}}{(b)_{n}}, \\
\mathcal{L}=\sum_{k=0}^{\infty} \frac{(a)_{k}(b)_{k}}{(1+a-b+i)_{k}(-1)^{k} k !}{ }_{2} F_{1}\left[\begin{array}{r}
-k, a+k \\
1+a-c
\end{array} ; 1\right] .
\end{gathered}
$$

Expressing ${ }_{2} F_{1}$ as a series, we have, after a little simplification

$$
\mathcal{L}=\sum_{k=0}^{\infty} \sum_{m=0}^{k} \frac{(a)_{k}(b)_{k}(-1)^{k}(-k)_{m}(a+k)_{m}}{(1+a-b+i)_{k} k !(1+a-c)_{m} m !} .
$$

Using the following identities

$$
\begin{aligned}
& (a)_{k}(a+k)_{m}=(a)_{k+m}, \\
& (-k)_{m}=\frac{(-1)^{m} k !}{(k-m) !}
\end{aligned}
$$


we have

$$
\mathcal{L}=\sum_{k=0}^{\infty} \sum_{m=0}^{k} \frac{(a)_{k+m}(b)_{k}(-1)^{k+m}}{(1+a-b+i)_{k}(1+a-c)_{m} m !(k-m) !} .
$$

Using a known result [7, p. 57, Eq. (2)]

$$
\sum_{n=0}^{\infty} \sum_{k=0}^{n} \mathcal{B}(k, n)=\sum_{n=0}^{\infty} \sum_{k=0}^{\infty} \mathcal{B}(k, n+k)
$$

we have

$$
\mathcal{L}=\sum_{k=0}^{\infty} \sum_{m=0}^{\infty} \frac{(a)_{k+2 m}(b)_{k+m}(-1)^{k}}{(1+a-b+i)_{k+m}(1+a-c)_{m} m ! k !} .
$$

Using the identities

$$
\begin{aligned}
& (a)_{k+2 m}=(a)_{2 m}(a+2 m)_{k}, \\
& (b)_{k+m}=(b)_{m}(b+m)_{k},
\end{aligned}
$$

we have

$$
\mathcal{L}=\sum_{m=0}^{\infty} \frac{(a)_{2 m}(b)_{m}}{(1+a-b+i)_{m}(1+a-c)_{m} m !} \sum_{k=0}^{\infty} \frac{(a+2 m)_{k}(b+m)_{k}(-1)^{k}}{(1+a-b+i+m)_{k} k !} .
$$

Summing up the inner series, we get

$$
\mathcal{L}=\sum_{m=0}^{\infty} \frac{(a)_{2 m}(b)_{m}}{(1+a-b+i)_{m}(1+a-c)_{m} m !}{ }_{2}^{2} F_{1}\left[\begin{array}{l}
a+2 m, b+m \\
1+a-b+i+m
\end{array} ;-1\right] .
$$

It is now easy to see that the ${ }_{2} F_{1}$ can be summed up by the known result (1.10) and after a little algebra, we easily arrive at the right-hand side of the Theorem 1. This completes the proof of the Theorem 1.

Corollary 2. (1) In Theorem 1, if we put $i=0$, we get after a little simplification, the classical Dixon's theorem (1.6).

(2) In Theorem 1, if we put $i=1$, we get, after some simplification, the following summation formula

$$
\begin{aligned}
& { }_{3} F_{2}\left[\begin{array}{c}
a, b, c \\
2+a-b, 1+a-c
\end{array} ;\right] \\
= & \frac{2^{-2 c+1} \Gamma(2+a-b) \Gamma(1+a-c)}{(b-1) \Gamma(a-2 b+2) \Gamma(a-b-c+2)} \\
& \times\left\{\frac{\Gamma\left(\frac{1}{2} a-b+3 / 2\right) \Gamma\left(\frac{1}{2} a-b-c+2\right)}{\Gamma\left(\frac{1}{2} a+\frac{1}{2}\right) \Gamma\left(\frac{1}{2} a-c+1\right)}+\frac{\Gamma\left(\frac{1}{2} a-b+1\right) \Gamma\left(\frac{1}{2} a-b-c+3 / 2\right)}{\Gamma\left(\frac{1}{2} a\right) \Gamma\left(\frac{1}{2} a-c+\frac{1}{2}\right)}\right\}
\end{aligned}
$$

provided $\Re(a-2 b-2 c)>1$.

Similarly, other results can also be obtained. 


\section{Generalization of Bailey's formula}

In this section, the result to be proved involving product of generalized hypergeometric series is given in the following theorem.

\section{Theorem 3.}

$$
\begin{aligned}
& \left.{ }_{0} F_{1}\left[\begin{array}{c}
- \\
\rho
\end{array}\right] \times{ }_{0} F_{1}\left[\begin{array}{c}
- \\
\rho+i
\end{array}\right]-x\right] \\
= & \frac{\Gamma\left(\frac{1}{2}\right) \Gamma(\rho) \Gamma(\rho+i)}{\Gamma\left(\rho+\frac{1}{2}(i+|i|)\right)} \sum_{m=0}^{\infty} \frac{(-1)^{m}(x)^{2 m}}{\left(\frac{1}{2}\right)_{m} 2^{2 m} m !\left(\frac{1}{2} \rho+\frac{1}{4}(i+|i|)\right)_{m}\left(\frac{1}{2} \rho+\frac{1}{4}(i+|i|)+\frac{1}{2}\right)_{m}} \\
& \times\left\{\frac{A_{i}{ }^{\prime}}{\Gamma\left(\rho+\frac{1}{2} i\right) \Gamma\left(\frac{1}{2}+\frac{1}{2} i-\left[\frac{1+i}{2}\right]\right)} \frac{\left(\frac{1}{2}-\frac{1}{2} i+\left[\frac{1+i}{2}\right]\right)_{m}}{\left(\rho+\frac{1}{2} i\right)_{m}}\right. \\
& \left.\quad+\frac{B_{i}{ }^{\prime}}{\Gamma\left(\rho+\frac{1}{2} i-\frac{1}{2}\right) \Gamma\left(\frac{1}{2} i-\left[\frac{i}{2}\right]\right)} \frac{\left(1-\frac{1}{2} i+\left[\frac{i}{2}\right]\right)_{m}}{\left(\rho+\frac{1}{2} i-\frac{1}{2}\right)_{m}}\right\} \\
+ & \frac{\Gamma\left(\frac{1}{2}\right) \Gamma(\rho) \Gamma(\rho+i)}{\Gamma\left(1+\rho+\frac{1}{2}(i+|i|)\right)} \sum_{m=0}^{\infty} \frac{(-1)^{m}(x)^{2 m+1}}{\left(\frac{3}{2}\right)_{m} 2^{2 m-1} m !\left(\frac{1}{2} \rho+\frac{1}{2}+\frac{1}{4}(i+|i|)\right)_{m}\left(\frac{1}{2} \rho+1+\frac{1}{4}(i+|i|)\right)_{m}} \\
& \times\left\{\frac{A_{i}^{\prime \prime}}{\Gamma\left(\rho+\frac{1}{2} i+\frac{1}{2}\right) \Gamma\left(\frac{1}{2} i-\left[\frac{1+i}{2}\right]\right)} \frac{\left(1-\frac{1}{2} i+\left[\frac{1+i}{2}\right]\right)_{m}}{\left(\rho+\frac{1}{2} i+\frac{1}{2}\right)_{m}}\right. \\
& \left.+\frac{B_{i}^{\prime \prime}}{\Gamma\left(\rho+\frac{1}{2} i\right) \Gamma\left(\frac{1}{2} i-\frac{1}{2}-\left[\frac{i}{2}\right]\right)} \frac{\left(\frac{3}{2}-\frac{1}{2} i+\left[\frac{i}{2}\right]\right)_{m}}{\left(\rho+\frac{1}{2} i\right)_{m}}\right\}
\end{aligned}
$$

for $i=0, \pm 1, \pm 2, \pm 3, \pm 4, \pm 5$.

The coefficients $A_{i}{ }^{\prime}$ and $B_{i}{ }^{\prime}$ can be obtained from the table of $A_{i}$ and $B_{i}$ by changing $a$ by $-2 m$ and $b$ by $1-\rho-2 m$ and the coefficients $A_{i}{ }^{\prime \prime}$ and $B_{i}{ }^{\prime \prime}$ can be obtained from the table of $A_{i}$ and $B_{i}$ by changing $a$ by $-2 m-1$ and $b$ by $-\rho-2 m$ respectively in $[6$, p. 298].

Proof. Denoting the left-hand side by $\mathcal{S}$, we have

$$
\mathcal{S}:={ }_{0} F_{1}\left[\begin{array}{c}
- \\
\rho
\end{array} ; x\right] \times{ }_{0} F_{1}\left[\begin{array}{c}
- \\
\rho+i
\end{array} ;-x\right] .
$$

Expressing both ${ }_{0} F_{1}$ as series, we have

$$
\mathcal{S}=\sum_{m=0}^{\infty} \sum_{n=0}^{\infty} \frac{(-1)^{n}}{(\rho)_{m}(\rho+i)_{n} m ! n !} x^{m+n},
$$

which, by virtue of the following well-known result [7, p. 56, Eq. (1)]

$$
\sum_{n=0}^{\infty} \sum_{k=0}^{\infty} \mathcal{B}(k, n)=\sum_{n=0}^{\infty} \sum_{k=0}^{n} \mathcal{B}(k, n-k),
$$


yields

$$
\mathcal{S}=\sum_{m=0}^{\infty} \sum_{n=0}^{m} \frac{(-1)^{n} x^{m}}{(\rho)_{m-n}(\rho+i)_{n}(m-n) ! n !} .
$$

Indeed, using the result (2.1) and after a little simplification, we get

$$
\mathcal{S}=\sum_{m=0}^{\infty} \frac{x^{m}}{(\rho)_{m} m !} \sum_{n=0}^{m} \frac{(-m)_{n}(1-\rho-m)_{n}(-1)^{n}}{(\rho+i)_{n} n !} .
$$

Summing up the inner series, we have

$$
\mathcal{S}=\sum_{m=0}^{\infty} \frac{x^{m}}{(\rho)_{m} m !}{ }_{2} F_{1}\left[\begin{array}{c}
-m, 1-\rho-m \\
\rho+i
\end{array} ;-1\right] .
$$

Separating even and odd powers of $x$, we have on using elementary identities [5]

$$
\begin{aligned}
& (a)_{2 m}=2^{2 m}\left(\frac{1}{2} a\right)_{m}\left(\frac{1}{2} a+\frac{1}{2}\right)_{m}, \\
& (a)_{2 m+1}=a 2^{2 m}\left(\frac{1}{2} a+\frac{1}{2}\right) m\left(\frac{1}{2} a+1\right)_{m}, \\
& (2 m) !=2^{2 m} m !\left(\frac{1}{2}\right)_{m} \\
& (2 m+1) !=2^{2 m} m !(3 / 2)_{m}
\end{aligned}
$$

$$
\begin{aligned}
\mathcal{S}= & \sum_{m=0}^{\infty} \frac{x^{2 m}}{2^{4 m}\left(\frac{1}{2} \rho\right)_{m}\left(\frac{1}{2} \rho+\frac{1}{2}\right)_{m}\left(\frac{1}{2}\right)_{m} m !}{ }^{2} F_{1}\left[\begin{array}{cc}
-2 m, 1-\rho-2 m \\
\rho+i
\end{array}\right. \\
& +\sum_{m=0}^{\infty} \frac{x^{2 m+1}}{\rho 2^{4 m}\left(\frac{1}{2} \rho+\frac{1}{2}\right)_{m}\left(\frac{1}{2} \rho+1\right)_{m}(3 / 2)_{m} m !}{ }^{2} F_{1}\left[\begin{array}{cc}
-2 m-1, & -\rho-2 m \\
\rho+i & ;-1
\end{array}\right] .
\end{aligned}
$$

Finally using generalized Kummer's summation formula in each ${ }_{2} F_{1}$ and after much simplification, we arrive at the right-hand side of the Theorem 3 . This completes the proof of Theorem 3 .

Corollary 4. We give some explicit special cases of Theorem 3.

For $i=0$

$$
{ }_{0} F_{1}\left[\begin{array}{c}
- \\
\rho
\end{array} x\right] \times{ }_{0} F_{1}\left[\begin{array}{c}
- \\
\rho
\end{array}-x\right]={ }_{0} F_{3}\left[\begin{array}{c}
- \\
\rho, \frac{1}{2} \rho, \frac{1}{2} \rho+\frac{1}{2} ;-\frac{x^{2}}{4}
\end{array} .\right.
$$

$$
\text { For } i=1
$$

$$
\begin{aligned}
& { }_{0} F_{1}\left[\begin{array}{c}
- \\
\rho
\end{array}\right] \times \times{ }_{0} F_{1}\left[\begin{array}{c}
- \\
\rho+1
\end{array}-x\right] \\
= & { }_{0} F_{3}\left[\begin{array}{c}
- \\
\rho, \frac{1}{2} \rho+\frac{1}{2}, \frac{1}{2} \rho+1
\end{array} ;-\frac{x^{2}}{4}\right]+\frac{x}{\rho(\rho+1)} 0 F_{3}\left[\rho+1, \frac{1}{2} \rho+1, \frac{1}{2} \rho+\frac{3}{2} ;-\frac{x^{2}}{4}\right] .
\end{aligned}
$$


For $i=-1$

(3.4)

$$
\begin{aligned}
& { }_{0} F_{1}\left[\begin{array}{c}
- \\
\rho
\end{array} ; x\right] \times{ }_{0} F_{1}\left[\begin{array}{c}
- \\
\rho-1
\end{array} ;-x\right] \\
& ={ }_{0} F_{3}\left[\rho-1, \frac{1}{2} \rho, \frac{1}{2} \rho+\frac{1}{2} ;-\frac{x^{2}}{4}\right]-\frac{x}{\rho(\rho-1)}{ }_{0} F_{3}\left[\rho, \frac{1}{2} \rho+\frac{1}{2}, \frac{1}{2} \rho+1 ;-\frac{x^{2}}{4}\right] \text {. }
\end{aligned}
$$

For $i=2$

(3.5)

$$
\begin{aligned}
& { }_{0} F_{1}\left[\begin{array}{c}
- \\
\rho
\end{array} ;\right] \times{ }_{0} F_{1}\left[\begin{array}{c}
- \\
\rho+2
\end{array}-x\right] \\
= & { }_{0} F_{3}\left[\rho, \frac{1}{2} \rho+1, \frac{1}{2} \rho+\frac{3}{2} ;-\frac{x^{2}}{4}\right]+\frac{2 x}{\rho(\rho+2)}{ }_{0} F_{3}\left[\rho+1, \frac{1}{2} \rho+2, \frac{1}{2} \rho+\frac{3}{2} ;-\frac{x^{2}}{4}\right] .
\end{aligned}
$$

For $i=-2$

(3.6)

$$
\begin{aligned}
& { }_{0} F_{1}\left[\begin{array}{c}
- \\
\rho
\end{array} ; x\right] \times{ }_{0} F_{1}\left[\begin{array}{c}
- \\
\rho-2
\end{array}-x\right] \\
= & { }_{0} F_{3}\left[\begin{array}{c}
- \\
\left.\rho-1, \frac{1}{2} \rho, \frac{1}{2} \rho+\frac{1}{2} ;-\frac{x^{2}}{4}\right]-\frac{2 x}{\rho(\rho-2)}{ }_{0} F_{3}\left[\rho-1, \frac{1}{2} \rho+\frac{1}{2}, \frac{1}{2} \rho+1 ;-\frac{x^{2}}{4}\right] .
\end{array}\right.
\end{aligned}
$$

For $i=3$

(3.7)

$$
\begin{aligned}
& { }_{0} F_{1}\left[\begin{array}{c}
- \\
\rho
\end{array} ; x\right] \times{ }_{0} F_{1}\left[\begin{array}{c}
- \\
\rho+3
\end{array} ;-x\right] \\
& ={ }_{0} F_{3}\left[\rho+1, \frac{1}{2} \rho+2, \frac{1}{2} \rho+\frac{3}{2} ;-\frac{x^{2}}{4}\right]+\frac{3 x}{\rho(\rho+3)}{ }_{0} F_{3}\left[\rho+2, \frac{1}{2} \rho+2, \frac{1}{2} \rho+\frac{5}{2} ;-\frac{x^{2}}{4}\right] \\
& +\frac{2 x^{2}}{\rho(\rho+1)(\rho+3)(\rho+4)}{ }_{0} F_{3}\left[\rho+2, \frac{1}{2} \rho+3, \frac{1}{2} \rho+\frac{5}{2} ;-\frac{x^{2}}{4}\right] \\
& -\frac{2 x^{3}}{\rho(\rho+1)(\rho+2)(\rho+3)(\rho+4)(\rho+5)}{ }_{0} F_{3}\left[\rho+3, \frac{1}{2} \rho+3, \frac{1}{2} \rho+\frac{7}{2} ;-\frac{x^{2}}{4}\right] .
\end{aligned}
$$


For $i=-3$

(3.8)

$$
\begin{aligned}
& { }_{0} F_{1}\left[\begin{array}{c}
- \\
\rho
\end{array} ; x\right] \times{ }_{0} F_{1}\left[\begin{array}{r}
- \\
\rho-3
\end{array} ;-x\right] \\
& ={ }_{0} F_{3}\left[\rho-2, \frac{1}{2} \rho, \frac{1}{2} \rho+\frac{1}{2} ;-\frac{x^{2}}{4}\right]-\frac{3 x}{\rho(\rho-3)}{ }_{0} F_{3}\left[\rho-1, \frac{1}{2} \rho+1, \frac{1}{2} \rho+\frac{1}{2} ;-\frac{x^{2}}{4}\right] \\
& +\frac{2 x^{2}}{\rho(\rho+1)(\rho-2)(\rho-3)}{ }_{0} F_{3}\left[\rho-1, \frac{1}{2} \rho+1, \frac{1}{2} \rho+\frac{3}{2} ;-\frac{x^{2}}{4}\right] \\
& -\frac{2 x^{3}}{\rho(\rho+1)(\rho+2)(\rho-1)(\rho-2)(\rho-3)}{ }_{0} F_{3}\left[\rho, \frac{1}{2} \rho+2, \frac{1}{2} \rho+\frac{3}{2} ;-\frac{x^{2}}{4}\right] \text {. }
\end{aligned}
$$

The result (3.2) is due to Bailey [2] and the results (3.3) to (3.8) are closely related to Bailey's result.

\section{Generalizations of the summations due to Ramanujan}

In this section, generalizations of the summations due to Ramanujan are given in the following theorem.

\section{Theorem 5.}

$$
\begin{aligned}
& 1-\left(\frac{1}{2}\right)^{2} \frac{1}{1+i} \frac{1}{1 !}+\left(\frac{1 \cdot 3}{2 \cdot 2}\right)^{2} \frac{1}{(1+i)(2+i)} \frac{1}{2 !}-\cdots+(-1)^{n} \frac{\left(\frac{1}{2}\right)_{n}\left(\frac{1}{2}\right)_{n}}{n !(1+i)_{n}}+\cdots \\
= & \frac{\pi \Gamma(1+i)}{\sqrt{2} \Gamma\left(\frac{1}{2}+i\right)}\left\{\frac{D_{i}}{\Gamma\left(\frac{3}{4}+\frac{1}{2} i\right) \Gamma\left(\frac{3}{4}+\frac{1}{2} i-\left[\frac{1+i}{2}\right]\right)}+\frac{D_{i}}{\Gamma\left(\frac{1}{4}+\frac{1}{2} i\right) \Gamma\left(\frac{1}{4}+\frac{1}{2} i-\left[\frac{i}{2}\right]\right)}\right\}
\end{aligned}
$$

for $i=0,1,2,3,4,5$.

The coefficients $C_{i}$ and $D_{i}$ can be obtained from the table of $A_{i}$ and $B_{i}$ by putting $a=b=\frac{1}{2}$ in [6, p. 298].

Theorem 6. For $\Re(x)>0$

$$
\begin{aligned}
& 1+\frac{(x-1)}{(x+1+i)}+\frac{(x-1)(x-2)}{(x+1+i)(x+2+i)}+\cdots+(-1)^{n} \frac{(1-x)_{n}}{(x+1+i)_{n}}+\cdots \\
= & \frac{\sqrt{\pi}(x+i) \Gamma(x)}{2}\left\{\frac{E_{i}}{\Gamma\left(x+\frac{1}{2}+\frac{1}{2} i\right) \Gamma\left(1+\frac{1}{2} i-\left[\frac{1+i}{2}\right]\right)}+\frac{F_{i}}{\Gamma\left(x+\frac{1}{2} i\right) \Gamma\left(\frac{1}{2}+\frac{1}{2} i-\left[\frac{i}{2}\right]\right)}\right\}
\end{aligned}
$$

for $i=0,1,2,3,4,5$.

The coefficients $E_{i}$ and $F_{i}$ can be obtained from the table of $A_{i}$ and $B_{i}$ by putting $a=1$ and $b=1-x$ respectively in [6]. 
Proof. The proof of the Theorems 5 and 6 are straight forward. It is easy to see that the left-hand side expression of the Theorem 5 corresponds to

$$
{ }_{2} F_{1}\left[\begin{array}{l}
\frac{1}{2}, \frac{1}{2} ;-1 \\
1+i
\end{array}\right]
$$

which is seen to be the special case of generalized Kummer's theorem (1.10), so it can easily be evaluated by (1.10) and we get the right-hand side of the Theorem 5. Similarly, it is easy to see that the left-hand side expression of the Theorem 6 corresponds to

$$
{ }_{2} F_{1}\left[\begin{array}{l}
1,1-x \\
1+x+i
\end{array} ;-1\right]
$$

which is also seen to be the special case of (1.10) and so it can easily be evaluated by (1.10) and we get the right-hand side of the Theorem 6 .

Corollary 7. If in our Theorem 5, we take $i=0,1,2$, we get the following summations.
(1) $1-\left(\frac{1}{2}\right)^{2}+\left(\frac{1 \cdot 3}{2 \cdot 4}\right)^{2}-\cdots=\frac{\sqrt{\pi}}{\sqrt{2} \Gamma^{2}(3 / 4)}$.
(2) $1-\frac{1}{2}\left(\frac{1}{2}\right)^{2}+\frac{1}{3}\left(\frac{1 \cdot 3}{2 \cdot 4}\right)^{2}-\cdots=\frac{\sqrt{2 \pi}}{\Gamma^{2}(3 / 4)}-\frac{2 \sqrt{2} \Gamma^{2}(3 / 4)}{\pi^{3 / 2}}$.
(3) $1-\frac{1}{3}\left(\frac{1}{2}\right)^{2}+\frac{1}{6}\left(\frac{1 \cdot 3}{2 \cdot 4}\right)^{2}-\cdots=\frac{16 \sqrt{2 \pi}}{9 \Gamma^{2}(3 / 4)}-\frac{16 \sqrt{2} \Gamma^{2}(3 / 4)}{3 \pi^{3 / 2}}$.

Similarly, other results can be obtained from our Theorem 5 .

Corollary 8. If in our Theorem 6, we take $i=0,1,2$, we get the following summations.

(1) $1+\frac{(x-1)}{(x+1)}+\frac{(x-1)(x-2)}{(x+1)(x+2)}+\cdots=\frac{\Gamma(3 / 2) \Gamma(x+1)}{\Gamma\left(x+\frac{1}{2}\right)}$.

(2) $1+\frac{(x-1)}{(x+2)}+\frac{(x-1)(x-2)}{(x+2)(x+3)}+\cdots=(x+1) \Gamma(x) \Gamma(3 / 2)\left\{\frac{1}{\Gamma\left(x+\frac{1}{2}\right)}-\frac{1}{\sqrt{\pi} \Gamma(x+1)}\right\}$.

(3) $1+\frac{(x-1)}{(x+3)}+\frac{(x-1)(x-2)}{(x+3)(x+4)}+\cdots=(x+2) \Gamma(x) \Gamma(3 / 2)\left\{\frac{(x+2)}{\Gamma\left(x+\frac{3}{2}\right)}-\frac{(3 x+4)}{\sqrt{\pi} \Gamma(x+2)}\right\}$.

\section{References}

[1] G. E. Andrews, R. Askey, and R. Roy, Special Functions, Encyclopedia of Mathematics and its Applications, 71. Cambridge University Press, Cambridge, 1999.

[2] W. N. Bailey, Product of generalized hypergeometric series, Proc. London Math. Soc. Ser. 228 (1928), 242-254.

[3] _ Generalized Hypergeometric Series, Cambridge Tracts in Mathematics and Mathematical Physics, No. 32 Stechert-Hafner, Inc., New York 1964.

[4] B. C. Berndt, Ramanujan's Notebooks, Part II, Springer-Verlag, New York, 1987.

[5] Y. S. Kim and A. K. Rathie, On an extension formulas for the triple hypergeometric series $X_{8}$ due to Exton, Bull. Korean Math. Soc. 44 (2007), no. 4, 743-751. 
[6] J. L. Lavoie, F. Grondin, and A. K. Rathie, Generalizations of Whipple's theorem on the sum of $a_{3} F_{2}$, J. Comput. Appl. Math. 72 (1996), no. 2, 293-300.

[7] E. D. Rainville, Special Functions, The Macmillan Company, New York, 1960.

\section{YONG SUP KIM}

Department of Mathematics Education

WONKWANG UNIVERSITY

IKSAN 570-749, KOREA

E-mail address: yspkim@wonkwang.ac.kr

ArJun K. Rathie

Department of Mathematics

Vedant College of Engineering and Technology

Tulsi-323021, Dist. Bundi, Rajasthan State, India

E-mail address: akrathie@rediffmail.com 